\title{
Ghost condensates in Yang-Mills theories in the Landau gauge
}

\author{
V.E.R. Lemes, M.S. Sarandy, and S.P. Sorella \\ UERJ - Universidade do Estado do Rio de Janeiro, \\ Rua São Francisco Xavier 524, 20550-013 Maracanã, Rio de Janeiro, Brazil.
}

October 27, 2018

\begin{abstract}
Ghost condensates of dimension two are analysed in pure $S U(N)$ Yang-Mills theories by combining the local composite operators technique with the algebraic BRST renormalization.
\end{abstract}




\section{Introduction}

The understanding of the nonperturbative effects which govern the infrared behavior of Yang-Mills theory is one of the most challenging issues in quantum field theory. Lattice results have provided evidences of the fact that the infrared regime of the theory is very different from the ultraviolet one, as expected by the asymptotic freedom. Simulations of the gluon propagator in the Landau gauge [1] and in the maximal Abelian gauge [2] display an infrared behavior compatible with the existence of a mass gap. Attempts to explain this behavior in the continuum have been given by taking into account Gribov's ambiguities and by using the Schwinger-Dyson equations, see for instance refs. 3, 4] for recent work on the subject. Nonperturbative effects are also at the origin of the existence of the quark condensate $\langle\bar{q} q\rangle$, responsible for the spontaneous breaking of the chiral symmetry, and of the pure gluon condensate $\left\langle\alpha F^{2}\right\rangle$.

Recently, much effort has been devoted to the study of condensates of dimension two built up with gauge fields and Faddeev-Popov ghosts. For instance, the gauge condensate $\left\langle A^{2}\right\rangle$ has a physical meaning in the Landau gauge [5, 6]. It plays a pivotal role in order to account for the discrepancy recently observed between the expected behavior of perturbation theory and the lattice results of the two and three point functions in the Landau gauge [7. 8. An effective potential for this condensate has been obtained in [9] by using the local composite operators (LCO) technique [10, 11]. This result shows that the vacuum of pure Yang-Mills theory favors a nonvanishing value of this condensate, which gives rise to a mass term for the gluons while contributing to the dimension four condensate $\left\langle\alpha F^{2}\right\rangle$ through the trace anomaly. Furthermore, as shown in the case of the compact $Q E D$ in $3 D$, the condensate $\left\langle A^{2}\right\rangle$ turns out to have a topological meaning [5], being argued to be suitable for detecting the presence of monopole configurations. A discussion of the relevance of $\left\langle A^{2}\right\rangle$ for confinement may be found in [12].

In addition to the gauge condensate $\left\langle A^{2}\right\rangle$, the ghost-antighost condensates $\langle\bar{c} c\rangle,\langle c c\rangle$ and $\langle\overline{c c}\rangle$ have also received great attention [13, 14, 15, 16, 17. in the context of the maximal Abelian gauge. This gauge, introduced in [18, 19], has provided evidences for monopoles condensation as well as for the Abelian dominance hypothesis [18, 20, which are key ingredients for the dual superconductivity mechanism of color confinement [21, 18. An important point to be noted here is that the maximal Abelian gauge is nonlinear. As a consequence, a quartic ghost interaction term must be necessarily in- 
cluded for renormalizability [22, 23]. This term gives rise to a nontrivial vacuum, corresponding to the existence of the aforementioned ghost condensates, which display rather interesting features. They modify the behavior of the ghost propagator in the infrared region [13, 14, 15] and lower the vacuum energy density, being interpreted as a low-energy manifestation of the trace anomaly 13, 14. Furthermore, $\langle\bar{c} c\rangle$ is believed to be part of a more general dimension two condensate, namely $\left(\frac{1}{2}\langle A A\rangle-\xi\langle c \bar{c}\rangle\right)$, where $\xi$ denotes the gauge parameter of the maximal Abelian gauge. This condensate has been proposed in [24] due to the property of being on-shell BRST invariant, and it is expected to provide effective masses for both off-diagonal gauge and ghost fields 24, 16, thus playing an important role for the Abelian dominance. It is worth remarking that the ghost condensates do not show up only in the maximal Abelian gauge, being present indeed in other nonlinear gauges as, for instance the so called Curci-Ferrari gauge [25, 26, 27. Also, the effective potential for the ghost-gluon condensate $\left(\frac{1}{2}\langle A A\rangle-\xi\langle c \bar{c}\rangle\right)$ in this gauge has recently been obtained [28].

The aim of this work is to analyze the ghost condensates in the Landau gauge. Although in this gauge there is no quartic ghost interaction, these condensates can be studied by means of the LCO technique, as improved by [10. 11. In particular, by combining the LCO technique with the BRST algebraic renormalization [29], we shall be able to obtain the effective potential for $\langle c c\rangle$ and $\langle\overline{c c}\rangle$. This result may signal a deeper physical meaning of these condensates.

The LCO formalism allows to obtain in a consistent way the effective potential for local composite operators. The method consists of introducing in the starting action the set of local composite operators to be studied coupled to suitable external sources. However, one has to be sure that all needed counterterms have been included. This requires the introduction of counterterms quadratic in the external sources. As shown in [10, 11, these quadratic terms can be dealt with by introducing a Hubbard-Stratonovich transformation allowing to obtain a renormalizable effective potential which is linear in the external sources, being thus compatible with the density energy interpretation of the effective potential. The method has already been successfully applied to the Gross-Neveu model [10] and to the $\lambda \phi^{4}$ and Coleman-Weinberg models [11]. In the latter case, a manifest gauge invariant two-loop effective potential has been obtained, according to the Nielsen identities.

It is worth underlining here that the LCO technique can be combined 
with the BRST algebraic renormalization [29] in order to obtain all needed counterterms. The algebraic renormalization allows indeed to write down a suitable set of Ward identities which determine, to all orders of perturbation theory, the most general counterterm depending on the fields and external sources needed for the renormalizability of a given set of local composite operators.

The paper is organized as follows. In Sect.2 we give a brief review of the LCO technique in the case of the condensate $\left\langle A^{2}\right\rangle$ for Yang-Mills in the Landau gauge. This example will allow us to show how the algebraic renormalization can be combined with the LCO method to provide a very powerful framework for the analysis of the effective potential. Sect.3 is devoted to the study of the ghost condensates $\langle c c\rangle$ and $\langle\overline{c c}\rangle$. In Sect.4 we present the conclusions.

\section{The LCO technique for the gauge conden- sate $\left\langle A_{\mu}^{a} A^{\mu a}\right\rangle$}

Let us begin with a brief review of the LCO technique [10, 11] in the case of the evaluation of the effective potential for the gauge condensate $\left\langle A_{\mu}^{a} A^{\mu a}\right\rangle$ in the Landau gauge 9 . It is known that we can give a meaning to the local composite operator $A_{\mu}^{a} A^{\mu a}$ in the Landau gauge. Indeed, due to the transversality condition $\partial_{\mu} A^{\mu a}=0$, the integrated mass dimension two operator $\int d^{4} x A_{\mu}^{a} A^{\mu a}$ is gauge invariant. It makes sense therefore to look at the condensate $\left\langle A_{\mu}^{a} A^{\mu a}\right\rangle$ in the Landau gauge.

In order to evaluate the effective potential for $A_{\mu}^{a} A^{\mu a}$ we introduce it in the starting action by means of an external source $j(x)$ of ghost number zero and dimension two [9], namely

$$
S_{\mathrm{YM}}+S_{\mathrm{gf}}+\int d^{4} x \frac{1}{2} j A_{\mu}^{a} A^{\mu a},
$$

where

$$
\begin{aligned}
S_{\mathrm{YM}} & =-\frac{1}{4} \int d^{4} x F^{a \mu \nu} F_{\mu \nu}^{a}, \\
S_{\mathrm{gf}} & =\int d^{4} x\left(b^{a} \partial_{\mu} A^{\mu a}+\bar{c}^{a} \partial^{\mu}\left(D_{\mu} c\right)^{a}\right),
\end{aligned}
$$


and

$$
\begin{aligned}
F_{\mu \nu}^{a} & =\partial_{\mu} A_{\nu}^{a}-\partial_{\nu} A_{\mu}^{a}+g f^{a b c} A_{\mu}^{b} A_{\nu}^{c}, \\
\left(D_{\mu} c\right)^{a} & =\partial_{\mu} c^{a}+g f^{a b c} A_{\mu}^{b} c^{c} .
\end{aligned}
$$

As it is well known, the gauge fixed action $\left(S_{\mathrm{YM}}+S_{\mathrm{gf}}\right)$ is left invariant by the BRST nilpotent transformations

$$
\begin{aligned}
s A_{\mu}^{a} & =-\left(D_{\mu} c\right)^{a}, \quad s c^{a}=\frac{g}{2} f^{a b c} c^{b} c^{c} \\
s \bar{c}^{a} & =b^{a} \quad, \quad s b^{a}=0 .
\end{aligned}
$$

However, before starting the computation, one has to be sure that the action (11) contains all needed counterterms. In other words we have to start with the most general action compatible with the invariances of the model and with the power-counting. Observe also that expression (11) with the inclusion of the coupling $j A^{2}$ is renormalizable by power-counting. The search for the most general action is easily handled by using the algebraic renormalization [29]. This will be the task of the next section.

\subsection{BRST characterization of the most general coun- terterm}

In order to characterize the most general action, including all needed counterterms, we proceed by introducing a BRST doublet [29, 30] of external sources $(j(x), \lambda(x))$, according to

$$
s \lambda=j, \quad s j=0,
$$

where $\lambda$ is an external source of dimension one and ghost number -1 .

\begin{tabular}{|l|c|c|}
\hline & $j$ & $\lambda$ \\
\hline Gh. number & 0 & -1 \\
\hline Dimension & 2 & 1 \\
\hline
\end{tabular}

It is apparent then that the quantity 


$$
S_{\mathrm{LCO}}=s \int d^{4} x\left(\lambda \frac{A^{2}}{2}+\frac{\varsigma}{2} \lambda j\right)=\int d^{4} x\left(\frac{1}{2} j A_{\mu}^{a} A^{\mu a}+\lambda A_{\mu}^{a} \partial^{\mu} c^{a}+\frac{\varsigma}{2} j^{2}\right),
$$

is BRST invariant. The meaning of the external source $\lambda(x)$ is also clear; it defines the composite operator $A_{\mu}^{a} \partial^{\mu} c^{a}$ which is the BRST variation of $A^{2} / 2$. Observe also that the BRST invariance and the power-counting naturally allow for the term $j^{2}=s(\lambda j)$. According now to [9, 10, 11, the parameter $\varsigma$ will be fixed by demanding that it depends on the gauge coupling, namely $\varsigma=\varsigma(g)$. The dependence of $\varsigma$ from $g$ can be computed order by order in the loop expansion

$$
\varsigma(g)=\varsigma_{0}+\hbar \varsigma_{1}+\hbar^{2} \varsigma_{2}+\ldots
$$

and is obtained from the renormalization group equations [9, 10, 11. This is a highly nontrivial requirement, which enables the LCO technique to capture nonperturbative effects [9, 10, 11]. In particular, the lowest order coefficient $\varsigma_{0}$ plays a fundamental role in order to obtain a nontrivial vacuum configuration for $\left\langle A^{2}\right\rangle$. In fact, as shown in [9], this coefficient is scheme independent ${ }^{1}$ and carries nonperturbative information, allowing for a nonvanishing value of $\left\langle A^{2}\right\rangle$.

Let us turn now to write down the Ward identities which will ensure the renormalizability of the model. We begin by observing that the action $\left(S_{\mathrm{YM}}+S_{\mathrm{gf}}+S_{\mathrm{LCO}}\right)$ is invariant under the BRST transformations (41) , (15)

$$
s\left(S_{\mathrm{YM}}+S_{\mathrm{gf}}+S_{\mathrm{LCO}}\right)=0 .
$$

Introducing also the external BRST invariant sources $\left(\Omega_{\mu}^{a}, L^{a}\right)$ coupled to the nonlinear variations of the fields $A_{\mu}^{a}$ and $c^{a}[29]$

$$
S_{\mathrm{ext}}=\int d^{4} x\left(-\Omega^{a \mu}\left(D_{\mu} c\right)^{a}+L^{a} \frac{g}{2} f^{a b c} c^{b} c^{c}\right),
$$

it turns out that the complete action

$$
\Sigma=S_{\mathrm{YM}}+S_{\mathrm{gf}}+S_{\mathrm{LCO}}+S_{\mathrm{ext}},
$$

obeys the following identities [29]

\footnotetext{
${ }^{1}$ We are indebted to D. Dudal for many valuable discussions on this important point.
} 
- the Slavnov-Taylor identity

$$
\begin{aligned}
& \mathcal{S}(\Sigma)=0 \\
& \mathcal{S}(\Sigma)=\int d^{4} x\left(\frac{\delta \Sigma}{\delta A_{\mu}^{a}} \frac{\delta \Sigma}{\delta \Omega^{a \mu}}+\frac{\delta \Sigma}{\delta c^{a}} \frac{\delta \Sigma}{\delta L^{a}}+b^{a} \frac{\delta \Sigma}{\delta \bar{c}^{a}}+j \frac{\delta \Sigma}{\delta \lambda}\right)
\end{aligned}
$$

- the Landau gauge condition and the antighost equation

$$
\frac{\delta \Sigma}{\delta b^{a}}=\partial_{\mu} A^{\mu a}, \quad \frac{\delta \Sigma}{\delta \bar{c}^{a}}+\partial^{\mu} \frac{\delta \Sigma}{\delta \Omega^{a \mu}}=0
$$

- the linearly broken integrated ghost equation Ward identity

$$
\int d^{4} x\left(\frac{\delta \Sigma}{\delta c^{a}}+g f^{a b c} \bar{c}^{b} \frac{\delta \Sigma}{\delta b^{c}}\right)=\Delta_{\mathrm{cl}}^{a},
$$

where $\Delta_{\mathrm{cl}}^{a}$ is the classical breaking

$$
\Delta_{\mathrm{cl}}^{a}=\int d^{4} x\left(g f^{a b c} \Omega^{b \mu} A_{\mu}^{c}-g f^{a b c} L^{b} c^{c}\right)
$$

We underline that the ghost equation Ward identity (13) is an important feature of the Landau gauge, which has allowed for an algebraic proof of several nonrenormalization results [31].

Let us now look at the most general counterterm depending on the external sources $(j, \lambda)$. Taking into account that $(j, \lambda)$ form a BRST doublet, it turns out that the most general expression depending on $(j, \lambda)$ with ghost number zero and dimension four is

$$
\begin{gathered}
S_{\mathrm{LCO}}^{\mathrm{count}}=s \int d^{4} x\left(a_{1} \lambda \frac{A^{2}}{2}+\frac{a_{2}}{2} \lambda j+a_{3} \lambda \bar{c}^{a} c^{a}\right) \\
=\int d^{4} x\left(a_{1} j \frac{A^{2}}{2}+a_{1} \lambda A_{\mu}^{a} \partial^{\mu} c^{a}+\frac{a_{2}}{2} j^{2}+a_{3} j \bar{c}^{a} c^{a}\right. \\
\left.-a_{3} \lambda b^{a} c^{a}+\frac{a_{3}}{2} \lambda \bar{c}^{a} f^{a b c} c^{b} c^{c}\right)
\end{gathered}
$$

However, $a_{3}=0$ due to the Landau ghost equation Ward identity (13) and to the gauge fixing condition and antighost equation (12). Thus 


$$
S_{\mathrm{LCO}}^{\mathrm{count}}=\int d^{4} x\left(a_{1} j \frac{A^{2}}{2}+a_{1} \lambda A_{\mu}^{a} \partial^{\mu} c^{a}+\frac{a_{2}}{2} j^{2}\right),
$$

which has precisely the same form as $S_{\mathrm{LCO}}$. The coefficient $a_{1}$ is related to the renormalization of the source $j$ and therefore to the renormalization of the local operator $A^{2}$. The coefficient $a_{2}$ can be associated to a renormalization of the parameter $\varsigma$. Its physical meaning is that of taking into account the divergences which appear in the correlator $\left\langle A^{2}(x) A^{2}(y)\right\rangle[9$. Finally we observe that, as expected by BRST invariance, the renormalization of the operator $A_{\mu}^{a} \partial^{\mu} c^{a}$ is related to that of $A^{2}$. Having characterized the most general local counterterm depending on the external sources $(j, \lambda)$, we are now ready to evaluate the one-loop effective potential for the condensate $\left\langle A^{2}\right\rangle$.

\subsection{The effective potential for $\left\langle A^{2}\right\rangle$}

The first step in order to study the condensate $\left\langle A^{2}\right\rangle$ is to analyse the generating functional $\mathcal{W}(j)$. Setting thus to zero the external sources $\Omega_{\mu}^{a}, L^{a}, \lambda$, we have

$$
\exp i \mathcal{W}(j)=\int[D \varphi] \exp i\left(S_{\mathrm{YM}}+S_{\mathrm{gf}}+\int d^{4} x\left(\frac{1}{2} j A_{\mu}^{a} A^{\mu a}+\frac{\varsigma}{2} j^{2}\right)\right)
$$

where $[D \varphi]$ denotes integration over all quantum fields $\left(A_{\mu}^{a}, b^{a}, \bar{c}^{a}, c^{a}\right)$. Taking the functional derivative of expression (17) we obtain

$$
\left.\frac{\delta \mathcal{W}(j)}{\delta j}\right|_{j=0}=\frac{1}{2}\left\langle A_{\mu}^{a} A^{\mu a}\right\rangle .
$$

In order to deal with the term $j^{2}$ we follow 9], introducing a HubbardStratonovich field $\sigma$ so that

$$
\left(\frac{1}{2} j A_{\mu}^{a} A^{\mu a}+\frac{\varsigma}{2} j^{2}\right)=\frac{1}{2}\left(\sqrt{\varsigma} j+\frac{1}{2 \sqrt{\varsigma}} A_{\mu}^{a} A^{\mu a}\right)^{2}-\frac{1}{8 \varsigma}\left(A_{\mu}^{a} A^{\mu a}\right)^{2},
$$

and 


$$
\frac{1}{2}\left(\sqrt{\varsigma} j+\frac{1}{2 \sqrt{\varsigma}} A_{\mu}^{a} A^{\mu a}\right)^{2} \Rightarrow-\frac{\sigma^{2}}{2 g^{2}}+\frac{1}{g} \sigma\left(\sqrt{\varsigma} j+\frac{1}{2 \sqrt{\varsigma}} A_{\mu}^{a} A^{\mu a}\right)
$$

As a consequence, for the functional generator $\mathcal{W}(j)$ we get

$$
\exp i \mathcal{W}(j)=\int[D \varphi] D \sigma \exp i\left(S(A, \sigma)+\int d^{4} x \frac{\sqrt{\varsigma}}{g} \sigma j\right)
$$

with

$$
S(A, \sigma)=S_{\mathrm{YM}}+S_{\mathrm{gf}}+\int d^{4} x\left(-\frac{\sigma^{2}}{2 g^{2}}+\frac{\sigma}{2 g \sqrt{\varsigma}} A_{\mu}^{a} A^{\mu a}-\frac{1}{8 \varsigma}\left(A_{\mu}^{a} A^{\mu a}\right)^{2}\right) .
$$

In particular,

$$
\left.\frac{\delta \mathcal{W}(j)}{\delta j}\right|_{j=0}=\frac{1}{2}\left\langle A_{\mu}^{a} A^{\mu a}\right\rangle=\frac{\sqrt{\varsigma}}{g}\langle\sigma\rangle_{S(A, \sigma)} .
$$

This is a very remarkable identity 9, stating that the condensate $\left\langle A^{2}\right\rangle$ is related to the nonvanishing expectation value of $\sigma$ evaluated with the new action $S(A, \sigma)$. Let us proceed thus with the computation of the effective potential for $\sigma$. We shall limit here to the one-loop order, which already displays all the nonperturbative features of the LCO technique.

\subsection{Evaluation of the one-loop effective potential}

In order to compute the effective potential for $\sigma$ at the one-loop order only the quadratic part of the action $S(A, \sigma)$ is relevant, namely

$$
S^{\text {quad }}(A, \sigma)=-\frac{\sigma^{2}}{2 g^{2}} \int d^{4} x+\frac{1}{2} \int d^{4} x A^{a \mu} \mathcal{M}_{\mu \nu}^{a b} A^{b \nu}
$$

with

$$
\mathcal{M}_{\mu \nu}^{a b}=\left[\left(\partial^{2} g_{\mu \nu}-\left(1-\frac{1}{\alpha}\right) \partial_{\mu} \partial_{\nu}\right)+\frac{\sigma}{g \sqrt{\varsigma}} g_{\mu \nu}\right] \delta^{a b}=\mathcal{A}_{\mu \nu} \delta^{a b}
$$


where $\sigma$ is now a constant field and the limit $\alpha \rightarrow 0$ has to be taken at the end in order to implement the Landau gauge. For the one-loop effective potential we have

$$
V^{\mathrm{eff}}(\sigma)=\frac{\sigma^{2}}{2 g^{2}}-\frac{i}{2} \operatorname{tr} \log \operatorname{det} \mathcal{M}_{\mu \nu}^{a b}
$$

From

$$
\operatorname{det} \mathcal{M}_{\mu \nu}^{a b}=\left(\operatorname{det} \mathcal{A}_{\mu \nu}\right)^{\left(N^{2}-1\right)}
$$

we have

$$
V^{\mathrm{eff}}(\sigma)=\frac{\sigma^{2}}{2 g^{2}}-\frac{i\left(N^{2}-1\right)}{2} \operatorname{tr} \log \operatorname{det} \mathcal{A}_{\mu \nu} .
$$

Making use of the dimensional regularization and recalling that

$$
\left.\log \operatorname{det} \mathcal{A}_{\mu \nu}\right|_{\alpha \rightarrow 0}=(d-1) \log \left(\partial^{2}+\frac{\sigma}{g \sqrt{\varsigma}}\right)+\text { const. }
$$

it follows

$$
V^{\mathrm{eff}}(\sigma)=\frac{\sigma^{2}}{2 g^{2}}-\frac{1}{2}\left(N^{2}-1\right) i \int \frac{d^{d} k}{(2 \pi)^{d}}(d-1) \log \left(-k^{2}+\frac{\sigma}{g \sqrt{\varsigma}}\right) .
$$

From

$i \int \frac{d^{d} k}{(2 \pi)^{d}} \log \left(\frac{\sigma}{g \sqrt{\varsigma}}-k^{2}\right)=\frac{1}{2(4 \pi)^{2}}\left(\frac{\sigma}{g \sqrt{\varsigma}}\right)^{2}\left[\frac{1}{\varepsilon}-\gamma+\frac{3}{2}-\log \frac{\sigma}{g \sqrt{\varsigma} 4 \pi \mu^{2}}\right]$

the one-loop effective potential in the $\overline{M S}$ scheme is found to be

$$
V^{\mathrm{eff}}(\sigma)=\frac{\sigma^{2}}{2 g^{2}}+\hbar \frac{3\left(N^{2}-1\right)}{64 \pi^{2}}\left(\frac{1}{g^{2} \varsigma}\right) \sigma^{2}\left(\log \frac{\sigma}{g \sqrt{\varsigma} \bar{\mu}^{2}}-\frac{5}{6}\right),
$$

where the presence of the loop expansion parameter $\hbar$ has been made explicit. Let us now proceed with the evaluation of the first term of the series (7)). To this purpose we require that the effective potential $V^{\text {eff }}(\sigma)$ obeys the renormalization group equation, namely

$$
\mu \frac{d V^{\mathrm{eff}}(\sigma)}{d \mu}=0+O\left(\hbar^{2}\right)
$$


To work out this condition the knowledge of the running of the field $\sigma$ is needed. This is easily achieved with the help of the relation between $\sigma$ and $\left\langle A^{2}\right\rangle$ of eq.(23), namely

$$
\left\langle\frac{A^{2}}{2}\right\rangle=\frac{\sqrt{\varsigma}}{g} \sigma
$$

A simple calculation gives

$$
\mu \partial_{\mu} \sigma=\hbar\left(\gamma_{A^{2}}^{(1)}+\frac{\beta_{g}^{(1)}}{g}-\frac{1}{2} \frac{\beta_{g}^{(1)}}{\varsigma_{0}} \frac{\partial \varsigma_{0}}{\partial g}\right) \sigma+O\left(\hbar^{2}\right)
$$

where $\gamma_{A^{2}}^{(1)}$ is the one-loop anomalous dimension of the local operator $A^{2}$ and, since $\varsigma=\varsigma(g)$,

$$
\mu \partial_{\mu} \varsigma=\beta_{g} \frac{\partial \varsigma}{\partial g}=\hbar \beta_{g}^{(1)} \frac{\partial \varsigma_{0}}{\partial g}+O\left(\hbar^{2}\right) .
$$

¿From eq. (33) it follows that

$$
\frac{1}{\varsigma_{0}}=\frac{16 \pi^{2}}{3\left(N^{2}-1\right)}\left(2 \gamma_{A^{2}}^{(1)}+2 \frac{\beta_{g}^{(1)}}{g}\right),
$$

from which one sees that $\varsigma_{0}$ is scheme independent. The explicit value of $\gamma_{A^{2}}^{(1)}$ can be found in [9, 32, 33] and turns out to be

$$
\gamma_{A^{2}}^{(1)}=\frac{g^{2} N}{16 \pi^{2}} \frac{35}{6}
$$

Therefore, recalling that

$$
\beta_{g}^{(1)}=-\frac{11 N}{3} \frac{g^{3}}{16 \pi^{2}}
$$

for $\varsigma_{0}$ one gets

$$
\varsigma_{0}=\frac{1}{g^{2}} \frac{9}{13} \frac{\left(N^{2}-1\right)}{N},
$$

in complete agreement with [9]. This expression displays the nonperturbative features of the LCO method [9], emphasized by the presence in eq.(40) of the factor $1 /\left(g^{2} N\right)$. 


\section{The ghost condensates}

Having given a brief summary of the LCO technique for the evaluation of $\left\langle A^{2}\right\rangle$, let us turn to the study of the ghost condensates. We shall consider the following local composite operators

$$
f^{a b c} c^{b} c^{c} \text { and } f^{a b c} \bar{c}^{b} \bar{c}^{c} .
$$

carrying ghost number 2 and -2 , respectively.

Following now the LCO procedure, we first introduce the two operators $f^{a b c} c^{b} c^{c}, f^{a b c} \bar{c}^{b} \bar{c}^{c}$ in the starting action by means of a set of external sources $\left(L^{a}, \tau^{a}, \eta^{a}\right)$ transforming as

$$
\begin{aligned}
& s \eta^{a}=\tau^{a}, \\
& s \tau^{a}=0, \\
& s L^{a}=0 .
\end{aligned}
$$

The quantum numbers of $\left(L^{a}, \tau^{a}, \eta^{a}\right)$ are displayed in the following table

\begin{tabular}{|l|l|l|l|}
\hline & $L^{a}$ & $\tau^{a}$ & $\eta^{a}$ \\
\hline Gh. number & -2 & 2 & 1 \\
\hline Dimension & 2 & 2 & 1 \\
\hline
\end{tabular}

Notice also that the sources $\left(\tau^{a}, \eta^{a}\right)$ form a BRST doublet. Moreover, according to the algebraic renormalization, the BRST invariant source $L^{a}$ is needed to define the nonlinear variation of the ghost $c^{a}$, which is $\left(g f^{a b c} c^{b} c^{c}\right) / 2$. In the present case, the BRST invariant part of the action depending on the external sources $\left(L^{a}, \tau^{a}, \eta^{a}\right)$ reads

$$
\begin{aligned}
S_{\mathrm{LCO}} & =s \int d^{4} x\left(L^{a} c^{a}-\frac{g}{2} \eta^{a} f^{a b c} \bar{c}^{b} \bar{c}^{c}+\varsigma \eta^{a} L^{a}\right) \\
& =\int d^{4} x\left(L^{a} \frac{g}{2} f^{a b c} c^{b} c^{c}-\frac{g}{2} \tau^{a} f^{a b c} \bar{c}^{b} \bar{c}^{c}+g \eta^{a} f^{a b c} b^{b} \bar{c}^{c}+\varsigma \tau^{a} L^{a}\right),
\end{aligned}
$$

where, as before, $\varsigma$ is a dimensionless parameter which will be fixed later on by demanding the compatibility with the renormalization group equations. Notice also that the presence of the term $\tau^{a} L^{a}$ is allowed by the power counting. For the complete action $\Sigma$ we have now 


$$
\Sigma=S_{\mathrm{YM}}+S_{\mathrm{m}}+S_{\mathrm{gf}}+S_{\mathrm{LCO}}+S_{\mathrm{ext}},
$$

where $S_{\mathrm{m}}$ stands for the fermionic matter action, which we include for completeness

$$
S_{\mathrm{m}}=\int d^{4} x \bar{\psi}^{i I} i \gamma^{\mu}\left(\partial_{\mu} \delta^{I J}-i g A_{\mu}^{a} T^{a I J}\right) \psi^{i J},
$$

where $T^{a I J}$ are the generators of $S U(N)$ in the fundamental representation, and $1 \leq i \leq n_{f}$ gives the number of fermions. Also, for the BRST transformations of the matter fields we have

$$
\begin{aligned}
s \psi^{i I} & =-i g c^{a} T^{a I J} \psi^{i J}, \\
s \bar{\psi}^{i I} & =-i g \bar{\psi}^{i J} T^{a J I} c^{a}, \\
s S_{\mathrm{m}} & =0 .
\end{aligned}
$$

Finally, introducing external sources $\Omega^{a \mu}, \bar{Y}^{i I}, Y^{i I}$, coupled to the nonlinear variations of the fields $A_{\mu}^{a}, \psi^{i I}$ and $\bar{\psi}^{i I}$, the external part of the complete action (45) reads

$$
S_{\mathrm{ext}}=\int d^{4} x\left[\Omega^{a \mu}\left(s A_{\mu}^{a}\right)+\bar{Y}^{i I}\left(s \psi^{i I}\right)+\left(s \bar{\psi}^{i I}\right) Y^{i I}\right] .
$$

It turns out that $\Sigma$ is constrained by the following identities:

- the Slavnov-Taylor identity

$$
\begin{gathered}
\mathcal{S}(\Sigma)=0 \\
\mathcal{S}(\Sigma)=\int d^{4} x\left[\frac{\delta \Sigma}{\delta A_{\mu}^{a}} \frac{\delta \Sigma}{\delta \Omega^{a \mu}}+\frac{\delta \Sigma}{\delta \bar{\psi}^{i I}} \frac{\delta \Sigma}{\delta Y^{i I}}+\frac{\delta \Sigma}{\delta \bar{Y}^{i I}} \frac{\delta \Sigma}{\delta \psi^{i I}}\right. \\
\left.+\left(\frac{\delta \Sigma}{\delta L^{a}}-\varsigma \tau^{a}\right) \frac{\delta \Sigma}{\delta c^{a}}+b^{a} \frac{\delta \Sigma}{\delta \bar{c}^{a}}+\tau^{a} \frac{\delta \Sigma}{\delta \eta^{a}}\right] .
\end{gathered}
$$

- the Landau gauge condition and the antighost equation

$$
\frac{\delta \Sigma}{\delta b^{a}}=\partial_{\mu} A^{\mu a}-g f^{a b c} \eta^{b} \bar{c}^{c},
$$




$$
\frac{\delta \Sigma}{\delta \bar{c}^{a}}+\partial^{\mu} \frac{\delta \Sigma}{\delta \Omega^{a \mu}}=g f^{a b c} \tau^{b} \bar{c}^{c}-g f^{a b c} \eta^{b} b^{c} .
$$

Notice that both the Landau gauge condition and the antighost equation gets modified by terms which are linear in the quantum fields, representing therefore classical breakings.

- the modified linearly broken integrated ghost equation Ward identity

$$
\int d^{4} x\left(\frac{\delta \Sigma}{\delta c^{a}}+g f^{a b c} c^{b} \frac{\delta \Sigma}{\delta b^{c}}+g f^{a b c} \eta^{b} \frac{\delta \Sigma}{\delta \tau^{c}}\right)=\Delta_{\mathrm{cl}}^{a},
$$

where $\Delta_{\mathrm{cl}}^{a}$ is the classical breaking

$$
\Delta_{\mathrm{cl}}^{a}=g \int d^{4} x\left[f^{a b c}\left(\Omega^{b \mu} A_{\mu}^{c}-L^{b} c^{c}+\varsigma \eta^{b} L^{c}\right)-i \bar{Y}^{i} T^{a} \psi^{i}+i \bar{\psi}^{i} T^{a} Y^{i}\right]
$$

Repeating now the same procedure as before, it is not difficult to prove that the complete action $\Sigma$ is stable with respect to radiative corrections. In other words $\Sigma$ is the most general action compatible with the Ward identities defining the model, up to a multiplicative renormalization of the fields, sources, the gauge coupling $g$ and the parameter $\varsigma$. In particular, concerning the allowed counterterms depending on the external sources $\left(L^{a}, \tau^{a}, \eta^{a}\right)$, it follows that the antighost equation (52) and the ghost Ward identity (531) imply the absence of counterterms of the type $L^{a} f^{a b c} c^{b} c^{c}, \quad \tau^{a} f^{a b c} \bar{c}^{b} \bar{c}^{c}$ and $\eta^{a} f^{a b c} b^{b} \bar{c}^{c}$ [31, 29, 33]. The only allowed counterterm in the external sources $\left(L^{a}, \tau^{a}, \eta^{a}\right)$ is given by $\tau^{a} L^{a}$. This counterterm corresponds to the renormalization of the LCO parameter $\varsigma$, stemming from the logarithmic divergences of the vacuum correlator $\left\langle c^{2}(x) \bar{c}^{2}(y)\right\rangle$.

\subsection{The effective potential for $\left\langle f^{a b c} c^{b} c^{c}\right\rangle$ and $\left\langle f^{a b c} \bar{c}^{b} \bar{c}^{c}\right\rangle$}

We can now begin the discussion of the effective potential for the local ghost operators $f^{a b c} c^{b} c^{c}$ and $f^{a b c} \bar{c}^{b} \bar{c}^{c}$. Let us start with the generating functional $\mathcal{W}(L, \tau)$. Setting thus to zero the external sources $\Omega_{\mu}^{a}, \eta^{a}, \bar{Y}^{i I}, Y^{i I}$ we have

$$
\begin{aligned}
\exp i \mathcal{W}(L, \tau)= & \int[D \varphi] \exp i\left(S_{\mathrm{YM}}+S_{\mathrm{m}}+S_{\mathrm{gf}}\right) \\
& \times \exp i\left[\int d^{4} x\left(L^{a} \frac{g f^{a b c} c^{b} c^{c}}{2}-\tau^{a} \frac{g f^{a b c} \bar{c}^{b} \bar{c}^{c}}{2}+\varsigma \tau^{a} L^{a}\right)\right]
\end{aligned}
$$


where $[D \varphi]$ denotes integration over all quantum fields $\left(A_{\mu}^{a}, b^{a}, \bar{c}^{a}, c^{a}, \psi^{i J}, \bar{\psi}^{i J}\right)$. Taking the functional derivative of expression (55) we obtain

$$
\left.\frac{\delta \mathcal{W}(L, \tau)}{\delta L^{a}}\right|_{L, \tau=0}=\left\langle\frac{g}{2} f^{a b c} c^{b} c^{c}\right\rangle
$$

and

$$
\left.\frac{\delta \mathcal{W}(L, \tau)}{\delta \tau^{a}}\right|_{L, \tau=0}=-\left\langle\frac{g}{2} f^{a b c} \bar{c}^{b} \bar{c}^{c}\right\rangle .
$$

In order to deal with the quadratic term $\tau^{a} L^{a}$ we introduce a pair of HubbardStratonovich fields $\left(\sigma^{a}, \bar{\sigma}^{a}\right)$ in the adjoint representation

\begin{tabular}{|l|l|l|}
\hline & $\sigma^{a}$ & $\bar{\sigma}^{a}$ \\
\hline Gh. number & 2 & -2 \\
\hline Dimension & 2 & 2 \\
\hline
\end{tabular}

so that

$$
L^{a} \frac{g}{2} f^{a b c} c^{b} c^{c}-\frac{g}{2} \tau^{a} f^{a b c} \bar{c}^{b} \bar{c}^{c}+\varsigma \tau^{a} L^{a} \Rightarrow \mathcal{L}_{\sigma \bar{\sigma}}
$$

where

$$
\begin{aligned}
& \mathcal{L}_{\sigma \bar{\sigma}}=-\frac{1}{g^{2}} \sigma^{a} \bar{\sigma}^{a}+\frac{\bar{\sigma}^{a}}{g \sqrt{\varsigma}}\left(\frac{g}{2} f^{a b c} c^{b} c^{c}\right)+\frac{\sqrt{\varsigma}}{g} \bar{\sigma}^{a} \tau^{a} \\
& -\frac{\sigma^{a}}{g \sqrt{\varsigma}}\left(\frac{g}{2} f^{a b c} \bar{c}^{b} \bar{c}^{c}\right)+\frac{\sqrt{\varsigma}}{g} \sigma^{a} L^{a}+\frac{1}{\varsigma}\left(\frac{g}{2} f^{a b c} c^{b} c^{c}\right)\left(\frac{g}{2} f^{a d e} \bar{c}^{d} \bar{c}^{e}\right) .
\end{aligned}
$$

With the introduction of the auxiliary fields $\left(\sigma^{a}, \bar{\sigma}^{a}\right)$, the generating functional $\mathcal{W}(L, \tau)$ becomes

$$
\exp i \mathcal{W}(L, \tau)=\int[D \varphi] D \sigma D \bar{\sigma} \exp i\left[S(A, \sigma, \bar{\sigma})+\int d^{4} x \frac{\sqrt{\varsigma}}{g}\left(\sigma^{a} L^{a}+\bar{\sigma}^{a} \tau^{a}\right)\right],
$$

with

$$
\begin{aligned}
S(A, \sigma, \bar{\sigma})=S_{\mathrm{YM}}+ & S_{\mathrm{m}}+S_{\mathrm{gf}}+\int d^{4} x\left(-\frac{1}{g^{2}} \sigma^{a} \bar{\sigma}^{a}+\frac{\bar{\sigma}^{a}}{g \sqrt{\varsigma}} \frac{g}{2} f^{a b c} c^{b} c^{c}\right. \\
& \left.-\frac{\sigma^{a}}{g \sqrt{\varsigma}}\left(\frac{g}{2} f^{a b c} \bar{c}^{b} \bar{c}^{c}\right)+\frac{1}{\varsigma}\left(\frac{g}{2} f^{a b c} c^{b} c^{c}\right)\left(\frac{g}{2} f^{a d e} \bar{c}^{d} \bar{c}^{e}\right)\right) .
\end{aligned}
$$


As in the case of the gauge condensate $\left\langle A^{2}\right\rangle$, differentiating $\mathcal{W}(L, \tau)$ with respect to $L$ and $\tau$, one obtains

$$
\begin{aligned}
& \left.\frac{\delta \mathcal{W}(L, \tau)}{\delta L^{a}}\right|_{L, \tau=0}=\frac{\sqrt{\varsigma}}{g}\left\langle\sigma^{a}\right\rangle_{S(A, \sigma, \bar{\sigma})}, \\
& \left.\frac{\delta \mathcal{W}(L, \tau)}{\delta \tau^{a}}\right|_{L, \tau=0}=\frac{\sqrt{\varsigma}}{g}\left\langle\bar{\sigma}^{a}\right\rangle_{S(A, \sigma, \bar{\sigma})},
\end{aligned}
$$

so that

$$
\left\langle\sigma^{a}\right\rangle=\frac{g^{2}}{2 \sqrt{\varsigma}}\left\langle f^{a b c} c^{b} c^{c}\right\rangle \quad, \quad\left\langle\bar{\sigma}^{a}\right\rangle=-\frac{g^{2}}{2 \sqrt{\varsigma}}\left\langle f^{a b c} \bar{c}^{b} \bar{c}^{c}\right\rangle .
$$

We see that the ghost condensates are related to the nonvanishing vacuum expectation value of $\sigma^{a}, \bar{\sigma}^{a}$ evaluated with the effective action $S(A, \sigma, \bar{\sigma})$ of equation (62) . Let us proceed thus with the evaluation of the one-loop effective potential for $\sigma^{a}, \bar{\sigma}^{a}$. To this order it is sufficient to consider only the terms of the action (62) which depend quadratically on the ghost fields $c^{a}, \bar{c}^{a}$, namely

$$
\begin{aligned}
S_{c \bar{c}}^{\text {quad }} & =\int d^{4} x\left(-\frac{1}{g^{2}} \sigma^{a} \bar{\sigma}^{a}+\bar{c}^{a} \partial^{2} c^{a}+\frac{\bar{\sigma}^{a}}{2 \sqrt{\varsigma}} f^{a b c} c^{b} c^{c}-\frac{\sigma^{a}}{2 \sqrt{\varsigma}} f^{a b c} \bar{c}^{b} \bar{c}^{c}\right) \\
& =\int d^{4} x\left[-\frac{1}{g^{2}} \sigma^{a} \bar{\sigma}^{a}+\frac{1}{2}\left(\begin{array}{cc}
\bar{c}^{a} & c^{a}
\end{array}\right) \mathcal{M}^{a b}\left(\begin{array}{c}
\bar{c}^{b} \\
c^{b}
\end{array}\right)\right],
\end{aligned}
$$

where $\mathcal{M}^{a b}$ denotes the $\left(N^{2}-1\right) \times\left(N^{2}-1\right)$ matrix

$$
\mathcal{M}^{a b}=\left(\begin{array}{ll}
-\frac{1}{\sqrt{\varsigma}} \sigma^{c} f^{c a b} & \partial^{2} \delta^{a b} \\
-\partial^{2} \delta^{a b} & \frac{1}{\sqrt{\varsigma}} \bar{\sigma}^{c} f^{c a b}
\end{array}\right) .
$$

For the one-loop effective potential we get

$$
V^{\mathrm{eff}}(\sigma, \bar{\sigma})=\frac{1}{g^{2}} \sigma^{a} \bar{\sigma}^{a}+\frac{i}{2} \operatorname{tr} \log \operatorname{det} \mathcal{M}^{a b},
$$

where $\sigma^{a}, \bar{\sigma}^{a}$ have to be considered constant fields. Let us focus on the case of $S U(2)$. In that case $f^{a b c}=\varepsilon^{a b c}\left(\varepsilon^{123}=1\right)$, and $\mathcal{M}^{a b}$ is a $6 \times 6$ matrix. After a straightforward computation one has

$$
V^{\mathrm{eff}}(\sigma, \bar{\sigma})=\frac{1}{g^{2}} \sigma^{a} \bar{\sigma}^{a}+i \int \frac{d^{d} k}{(2 \pi)^{d}} \log \left(\left(k^{2}\right)^{2}+\frac{\sigma^{a} \bar{\sigma}^{a}}{\varsigma}\right) .
$$


¿From

$$
\int \frac{d^{d} k}{(2 \pi)^{d}} \log \left(\left(k^{2}\right)^{2}+\varphi^{2}\right)=\frac{i}{32 \pi^{2}} \varphi^{2}\left(-\frac{1}{\varepsilon}-2 \gamma+2 \log 4 \pi-\log \frac{\varphi^{2}}{\mu^{4}}+3\right),
$$

the effective potential in the $\overline{M S}$ scheme is found to be

$$
V^{\mathrm{eff}}(\sigma, \bar{\sigma})=\frac{1}{g^{2}} \sigma^{a} \bar{\sigma}^{a}+\frac{1}{32 \pi^{2}} \frac{1}{\varsigma_{0}} \sigma^{a} \bar{\sigma}^{a}\left(\ln \frac{\sigma^{a} \bar{\sigma}^{a}}{\varsigma_{0} \bar{\mu}^{4}}-3\right) .
$$

The minimum of the effective potential (170) is given by the condition

$$
\sigma_{\min }^{a} \bar{\sigma}_{\min }^{a}=\varsigma_{0} \bar{\mu}^{4} e^{2} \exp \left(-\frac{32 \pi^{2} \varsigma_{0}}{g^{2}}\right),
$$

which, up to one-loop order, is physically consistent for $\varsigma_{0}>0$. Setting

$$
\sigma^{a}=\sigma \delta^{a 3}, \quad \bar{\sigma}^{a}=\bar{\sigma} \delta^{a 3},
$$

we obtain

$$
V^{\mathrm{eff}}(\sigma, \bar{\sigma})=\frac{1}{g^{2}} \sigma \bar{\sigma}+\hbar \frac{1}{32 \pi^{2}} \frac{1}{\varsigma_{0}} \sigma \bar{\sigma}\left(\ln \frac{\sigma \bar{\sigma}}{\varsigma_{0} \bar{\mu}^{4}}-3\right) .
$$

Let us now evaluate $\varsigma_{0}$. The first step is to obtain the running of the fields $\sigma$, $\bar{\sigma}$. This task is easily accomplished by recalling that, in the Landau gauge, the Faddeev-Popov ghosts renormalize as 31, 33.

$$
\begin{aligned}
& c_{b}=Z_{c}^{1 / 2} c, \\
& \bar{c}_{b}=Z_{c}^{1 / 2} \bar{c}
\end{aligned}
$$

with

$$
Z_{c}=Z_{g}^{-1} Z_{A}^{-1 / 2}
$$

where

$$
g_{b}=Z_{g} g, \quad A_{b}=Z_{A}^{1 / 2} A .
$$

where the $g_{b}, A_{b}, \bar{c}_{b}, c_{b}$ denote the bare coupling constant and fields. From the absence [31, 33] of the counterterms $L^{a} f^{a b c} c^{b} c^{c}, \tau^{a} f^{a b c} \bar{c}^{b} \bar{c}^{c}$ it follows

$$
\begin{aligned}
L_{b} g_{b} c_{b}^{2} & =L g c^{2} \\
\tau_{b} g_{b} \bar{c}_{b}^{2} & =\tau g \bar{c}^{2}
\end{aligned}
$$


so that

$$
\begin{aligned}
L_{b} & =Z_{A}^{1 / 2} L, \quad \gamma_{L}=\gamma_{A} \\
\tau_{b} & =Z_{A}^{1 / 2} L, \quad \gamma_{\tau}=\gamma_{A},
\end{aligned}
$$

where $\gamma_{A}$ is the anomalous dimension of the gauge field $A_{\mu}^{a}$. Therefore, making use of the equations (64), for the running of $\sigma, \bar{\sigma}$ we find

$$
\begin{aligned}
& \mu \partial_{\mu} \sigma=\hbar\left(\gamma_{A}^{(1)}+\frac{\beta_{g}^{(1)}}{g}-\frac{1}{2} \frac{\beta_{g}^{(1)}}{\varsigma_{0}} \frac{\partial \varsigma_{0}}{\partial g}\right) \sigma+O\left(\hbar^{2}\right), \\
& \mu \partial_{\mu} \bar{\sigma}=\hbar\left(\gamma_{A}^{(1)}+\frac{\beta_{g}^{(1)}}{g}-\frac{1}{2} \frac{\beta_{g}^{(1)}}{\varsigma_{0}} \frac{\partial \varsigma_{0}}{\partial g}\right) \bar{\sigma}+O\left(\hbar^{2}\right) .
\end{aligned}
$$

Requiring now the renormalization group invariance of the effective potential $V^{\text {eff }}(\sigma, \bar{\sigma})$, namely

$$
\mu \frac{d V^{\mathrm{eff}}(\sigma, \bar{\sigma})}{d \mu}=0+O\left(\hbar^{2}\right)
$$

for the coefficient $\varsigma_{0}$ one obtains

$$
\frac{1}{8 \pi^{2}} \frac{1}{\varsigma_{0}}=\frac{2}{g^{2}} \gamma_{A}^{(1)}
$$

Finally, recalling that in the case of $S U(2)$ 32

$$
\begin{aligned}
& \beta_{g}^{(1)}=-\left(\frac{22}{3}-\frac{2}{3} n_{f}\right) \frac{g^{3}}{16 \pi^{2}}, \\
& \gamma_{A}^{(1)}=\left(-\frac{13}{3}+\frac{2}{3} n_{f}\right) \frac{g^{2}}{16 \pi^{2}},
\end{aligned}
$$

it follows from (83) that

$$
\frac{1}{\varsigma_{0}}=\left(\frac{2 n_{f}-13}{3}\right)
$$

completing therefore the evaluation of the one-loop effective potential for the ghost condensates. A few remarks are now in order.

- By combining the LCO technique with the BRST algebraic renormalization we have been able to obtain the one-loop effective potential for the ghost condensates. By construction, the effective potential $V^{\mathrm{eff}}(\sigma, \bar{\sigma})$ obeys the renormalization group equation. 
- From the equations (82), (83) we observe that when the number of fermions $n_{f}$ is chosen so that

$$
6<n_{f}<11
$$

we get a positive value for $\varsigma_{0}$, while keeping $\beta_{g}<0$, so as to ensure asymptotic freedom. This result gives an evidence for the existence of these condensates. We also observe that even for $n_{f} \leq 6$ the effective potential $V^{\text {eff }}(\sigma, \bar{\sigma})$ seems to admit a nonvanishing minimum. However, in this case higher loops contributions are needed in order to obtain a deeper understanding of that minimum 34 .

- In the present work we have considered the charged ghost polynomials $f^{a b c} c^{b} c^{c}$ and $f^{a b c} \bar{c}^{b} \bar{c}^{c}$. The same procedure could have been applied to study the ghost polynomial $f^{a b c} c^{b} \bar{c}^{c}$, which has vanishing FaddeevPopov charge. As underlined in [17, these operators correspond to different channels for the ghost condensation, being related to the generators of the global symmetry $S L(2, R)$ present in the Landau gauge. It is worth mentioning that the existence of different channels for the ghost condensation has an analogy in superconductivity, known as the BCS ${ }^{2}$ versus the Overhauser ${ }^{3}$ effect [35]. In the present case the FaddeevPopov charged condensates $\left\langle f^{a b c} c^{b} c^{c}\right\rangle,\left\langle f^{a b c} \bar{c}^{b} \bar{c}^{c}\right\rangle$ would correspond to the BCS channel, while $\left\langle f^{a b c} c^{b} \bar{c}^{c}\right\rangle$ to the Overhauser channel. Although our aim here is that of showing how to construct the effective potential for the ghost condensation in the Landau gauge, we remark that both channels could be studied simultaneously in order to decide which one has the lowest energy 34.

- Finally, let us give the ghost propagators in the condensed vacuum, namely

$$
\begin{aligned}
\left\langle\bar{c}^{\alpha}(p) c^{\beta}(-p)\right\rangle & =-i \frac{p^{2} \delta^{\alpha \beta}}{\left(p^{2}\right)^{2}+v^{4}} \quad, \quad \alpha, \beta=1,2 \\
\left\langle\bar{c}^{3}(p) c^{3}(-p)\right\rangle & =-\frac{i}{p^{2}} .
\end{aligned}
$$

\footnotetext{
${ }^{2}$ Particle-particle and hole-hole pairing.

${ }^{3}$ Particle-hole pairing.
} 
where

$$
v^{4}=\frac{\bar{\sigma}_{\min } \sigma_{\min }}{2 \varsigma_{0}}
$$

One see thus that the off-diagonal propagators get deeply modified in the infrared region, the ghost condensation $\bar{\sigma}_{\min } \sigma_{\min }$ providing the infrared cutoff.

\section{Conclusion}

In this work the one-loop effective potential for the ghost condensates $\langle c c\rangle$ and $\langle\overline{c c}\rangle$ has been obtained in the Landau gauge by combining the local composite operators technique (LCO) with the BRST algebraic renormalization. Our results might signal a deeper physical meaning of these condensates, which have been proven to exist in other gauges [13, 14, 15, 25, 26, 27. The existence of nonvanishing ghost condensates in the Landau gauge can also be justified by symmetry breaking considerations. Although the Landau gauge does not possess a quartic ghost self-interaction, it shares an important feature with the nonlinear gauge considered in [25, 26, 27]. Both gauges display indeed a global $S L(2, R)$ symmetry 36, present in $S U(N)$ Yang-Mills for any value of $N$. It is very interesting to point out that the $S L(2, R)$ symmetry is also present in the maximal Abelian gauge in the case of $S U(2)$, as underlined by [13]. Recent investigations have proven that $S L(2, R)$ is indeed present in this gauge for a generic $S U(N)$ [17]. Therefore, the existence of ghost condensates in these gauges can be seen as the manifestation of a very general feature, i.e. the dynamical symmetry breaking of $S L(2, R)$.

As one can see from the expressions (86), the ghost condensates modify the behavior of the propagator in the infrared region. Recent lattice simulations for the propagator in the Landau gauge are nicely reproduced by a form factor $F\left(p^{2}\right)$ whose infrared behavior is given by [1]

$$
\sum_{\mu a}\left\langle A_{\mu}^{a}(p) A_{\mu}^{a}(-p)\right\rangle=\frac{F\left(p^{2}\right)}{p^{2}}
$$

with

$$
F\left(p^{2}\right)=\mathcal{N} \frac{p^{2}}{p^{2}+m_{1}^{2}}\left[\frac{1}{p^{4}+m_{2}^{4}}+\frac{s}{\left(\log \left(m_{L}^{2}+p^{2}\right)\right)^{13 / 22}}\right],
$$


where $\mathcal{N}, s, m_{1}, m_{2}$ and $m_{L}$ are fitting parameters [1] whose values are $\mathcal{N}=8.113, s=0.32, m_{1}=0.64 \mathrm{GeV}, m_{2}=1.31 \mathrm{GeV}$ and $m_{L}=1.23 \mathrm{GeV}$. Although a fully theoretical analytic derivation of this behavior is still lacking, it is tempting to argue that both the gauge $\left\langle A^{2}\right\rangle$ and the ghost condensates $\langle c c\rangle,\langle\overline{c c}\rangle,\langle c \bar{c}\rangle$ could give some useful insights in order to improve our knowledge of the infrared region. Needless to say, the gauge and the ghost propagators mix nontrivially in the Schwinger-Dyson equations for the Landau gauge.

Finally, we point out that the LCO technique can be combined with the BRST algebraic renormalization to provide a powerful framework for analysing the quantum properties of local composite operators. Particular attention will be devoted to the condensate $\left(\frac{1}{2}\langle A A\rangle-\xi\langle c \bar{c}\rangle\right)$ in the maximal Abelian gauge. This condensate is BRST on-shell invariant and it is expected to provide effective masses for both off-diagonal gauge and ghost fields [24. 16], a point of great relevance for the Abelian dominance.

\section{Acknowledgments}

The Conselho Nacional de Desenvolvimento Científico e Tecnológico CNPqBrazil, the Fundação de Amparo a Pesquisa do Estado do Rio de Janeiro (Faperj) and the SR2-UERJ are acknowledged for the financial support. We are grateful to D. Dudal and to M. Picariello for fruitful discussion.

\section{References}

[1] K. Langfeld, H. Reinhardt and J. Gattnar, Nucl.Phys. B621 (2002) 131.

[2] K. Amemiya and H. Suganuma, Phys.Rev. D60 (1999) 114509.

[3] D. Zwanziger, Time independent stochastic quantization, DS equations, and infrared critical exponents in QCD, hep-th/0206053,

D. Zwanziger, Phys.Rev. D65 (2002) 094039.

[4] C. Lerche and L. von Smekal, Phys.Rev. D65 (2002) 125006;

R. Alkofer and L. von Smekal, Phys.Rept. 353 (2001) 281. 
[5] F.V. Gubarev, L. Stodolsky and V.I. Zakharov, Phys. Rev. Lett. 86 (2001) 2220.

[6] L. Stodolsky, P. van Baal, V.I. Zakharov, Phys. Lett. B552 (2003) 214.

[7] Ph. Boucaud et al., JHEP 0004 (2000) 006;

Ph. Boucaud, A. Le Yaouanc, J.P. Leroy, J. Micheli, O. Pène, J. Rodríguez-Quintero, Phys. Lett. B493 (2000) 315;

Ph. Boucaud, J.P. Leroy, A. Le Yaouanc, J. Micheli, O. Pène, J. Rodríguez-Quintero, Phys. Rev. D63 (2001) 114003;

Ph. Boucaud, J.P. Leroy, A. Le Yaouanc, J. Micheli, O. Pène, F. De Soto, A. Donini, H. Moutarde, J. Rodríguez-Quintero, Phys.Rev. D66 (2002) 034504.

[8] F.V. Gubarev and V.I. Zakharov, Phys. Lett. B501 (2001) 28.

[9] H. Verschelde, K. Knecht, K. Van Acoleyen, M. Vanderkelen, Phys. Lett. B516 (2001) 307.

[10] H. Verschelde, Phys.Lett. B351 (1995) 242;

H. Verschelde, S. Schelstraete, M. Vanderkelen, Z.Phys. C76 (1997) 161.

[11] K. Knecht and H. Verschelde, Phys. Rev. D64 (2001) 08500610.

[12] K.-I. Kondo and T. Ima, A Confining String Theory Derivable from Yang-Mills Theory due to a Novel Vacuum Condensate, hep-th/0206173.

[13] M. Schaden, Mass Generation in Continuum SU(2) Gauge Theory in Covariant Abelian Gauges, hep-th/9909011, Mass Generation, Ghost Condensation and Broken Symmetry: SU(2) in Covariant Abelian Gauges, talk given at Confinement IV, Vienna, 2000, hep-th/0108034; SU(2) Gauge Theory in Covariant (Maximal) Abelian Gauges, talk presented at Vth Workshop on QCD, Villefranche, 2000, hep-th/0003030.

[14] K.-I. Kondo and T. Shinohara, Phys. Lett. B491 (2000) 263.

[15] V.E.R. Lemes, M.S. Sarandy and S.P. Sorella, Ghost Number Dynamical Symmetry Breaking in Yang-Mills Theories in the Maximal Abelian Gauge, hep-th/0206251. 
[16] D. Dudal and H. Verschelde, On ghost condensation, mass generation and Abelian dominance in the Maximal Abelian Gauge, hep-th/0209025.

[17] D. Dudal, V.E.R. Lemes, M. Picariello, M. S. Sarandy, S. P. Sorella, H. Verschelde, JHEP 0212 (2002) 008.

[18] G. 't Hooft, Nucl. Phys. B190 [FS3] (1981) 455.

[19] A. Kronfeld, G. Schierholz and U.-J. Wiese, Nucl. Phys. B293 (1987) 461 ;

A. Kronfeld, M. Laursen, G. Schierholz and U.-J. Wiese, Phys. Lett. B198 (1987) 516.

[20] T. Susuki and I. Yotsuyanagi, Phys. Rev. D42 (1990) 4257.

[21] Y. Nambu, Phys. Rev. D10 (1974) 4262;

G. 't Hooft, High Energy Physics EPS Int. Conference, Palermo 1975, ed. A. Zichichi;

S. Mandelstam, Phys. Rept. 23 (1976) 245.

[22] H. Min, T. Lee and P.Y. Pac, Phys. Rev. D32 (1985) 440.

[23] A.R. Fazio, V.E.R. Lemes, M.S. Sarandy and S.P. Sorella, Phys. Rev. D64 (2001) 085003.

[24] K.-I. Kondo, Phys. Lett. B514 (2001) 335; K.-I.Kondo, T.Murakami, T.Shinohara, T.Imai, Phys. Rev. D65 (2002) 085034.

[25] K.-I. Kondo, Spontaneous breakdown of BRST supersymmetry due to ghost condensation in QCD, hep-th/0103141.

[26] A.R. Fazio, V.E.R. Lemes, M. Picariello, M.S. Sarandy and S.P. Sorella, Ghost condensates in Yang-Mills theories in nonlinear gauges, hep-th/0210036.

[27] H. Sawayanagy, Phys. Rev. D67 (2003) 045002.

[28] D. Dudal, H. Verschelde, V. E. R. Lemes, M. S. Sarandy, S. P. Sorella, M. Picariello, Gluon-ghost condensate of mass dimension 2 in the CurciFerrari gauge, hep-th/0302168 
[29] O. Piguet and S.P. Sorella, Algebraic Renormalization, Monograph series m28, Springer Verlag, 1995.

[30] G. Barnich, F. Brandt and M. Henneaux, Phys. Rept. 338 (2000) 439.

[31] A. Blasi, O. Piguet and S.P. Sorella, Nucl. Phys. B356 (1991) 154;

O. Piguet and S.P. Sorella, Nucl. Phys. B381 (1992) 373.

[32] J.A. Gracey, Phys.Lett. B552 (2003) 101;

[33] D. Dudal, H. Verschelde, S.P. Sorella, Phys.Lett. B555 (2003) 126.

[34] D. Dudal, H. Verschelde, V.E.R. Lemes, M.S. Sarandy, S.P. Sorella, M. Picariello, work in progress.

[35] B.-Y. Park, M. Rho, A. Wirzba, I. Zahed, Phys. Rev. D62 (2000) 034015.

[36] R. Delbourgo and P.D. Jarvis, J.Phys. A15 (1982) 611;

L. Baulieu and J. Thierry-Mieg, Nucl.Phys. B197 (1982) 477;

I. Ojima, Z.Phys. C13 (1982) 173;

F. Delduc and S.P. Sorella, Phys.Lett. B231 (1989) 408. 